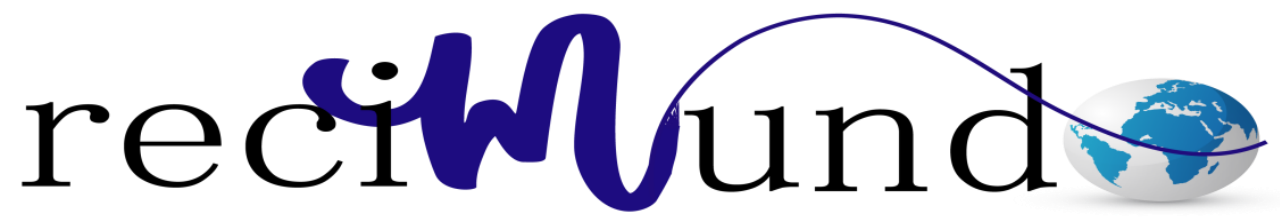

Revista Científica Mundo de la Investigación y el Conocimiento

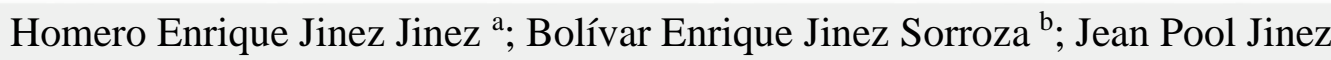

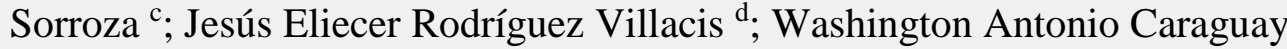

Ambuludi ${ }^{e}$; Marco Vinicio Sotomayor Sánchez ${ }^{\mathrm{f}}$

Computación en la nube

Revista Científica Mundo de la Investigación y el Conocimiento. Vol. 2 núm., 1, febrero, ISSN: 2588-073X, 2018, pp. 703-715

DOI: $\underline{10.26820 / \text { recimundo/2.1.2018.703-715 }}$

Editorial Saberes del Conocimiento

Recibido: 05/12/2017

Aceptado: 10/02/2018

a. Laboratorio de análisis clínico "DAYANA"; hejinezjinez@ hotmail.com

b. Laboratorio de análisis clínico "DAYANA"; kikejinez@gmail.com

c. Universidad Espíritu Santo; jjinez@uees.edu.ec

d. Universidad Espíritu Santo; jesusrodriguez@uees.edu.ec

e. Universidad Espíritu Santo; wcaraguay@uees.edu.ec

f. Universidad Espíritu Santo; mvinicio@uees.edu.ec 


\section{Computación en la nube}

Vol. 2, núm. 1., (2018)

Homero Enrique Jinez Jinez; Bolívar Enrique Jinez Sorroza; Jean Pool Jinez Sorroza; Jesus Eliecer Rodríguez Villacis; Washington Antonio Caraguay Ambuludi; Marco Vinicio Sotomayor Sánchez

\section{RESUMEN}

El Cloud Computing para nosotros, usuarios comunes y corrientes, probablemente sea ya cosa de todos los días. Todos usamos Gmail, Hotmail, o cualquier otro servicio de correo electrónico, ¿no? Entonces ya estamos utilizando de alguna manera el Cloud Computing; y es que, poco a poco, el navegador, que antes servía para eso, para navegar por la Web y poco más, se está convirtiendo en nuestro nuevo sistema operativo, dada la cantidad de usos que le damos. ¿Alguna vez ha pensado en todo lo que el navegador puede hacer? Antes, si queríamos mandar un mensaje, teníamos que abrir un programa específico; para chatear, otro; y para escuchar música, teníamos que abrir un reproductor de medios. Hoy, el navegador es capaz de englobar todo eso. ¿Para qué instalar entonces programas o aplicaciones en nuestros ordenadores si podemos obtener los mismos servicios sin la necesidad de hacerlo? Esto es el Cloud Computing para nosotros, los usuarios comunes. Muchas de las aplicaciones que utilizábamos han dado el salto a la nube, donde son totalmente independientes del sistema operativo que esté accediendo a ellas, y con una gran ventaja: que todo es portátil. Pero es mucho más que eso, y es que el Cloud Computing ha transformado de forma radical el modelo de negocio, tanto de pymes como de grandes empresas, y sus estrategias al disponer de una nueva herramienta que permite acelerar la innovación de procesos y reducir costes.

Palabras claves: Servidor, Internet, Aplicaciones, Red, Navegador. 


\section{Computación en la nube}

Vol. 2, núm. 1., (2018)

Homero Enrique Jinez Jinez; Bolívar Enrique Jinez Sorroza; Jean Pool Jinez Sorroza; Jesus Eliecer Rodríguez Villacis; Washington Antonio Caraguay Ambuludi; Marco Vinicio Sotomayor

Sánchez

\section{ABSTRACT}

The Cloud Computing for us, ordinary users, probably is already something of every day. We all use Gmail, Hotmail, or any other email service, right? So we are already using Cloud Computing in some way; and is that, little by little, the browser, which used to be used for that, to browse the Web and little else, is becoming our new operating system, given the number of uses we give it. Have you ever thought about everything the browser can do? Before, if we wanted to send a message, we had to open a specific program; to chat, another; and to listen to music, we had to open a media player. Today, the browser is able to encompass all that. Why then install programs or applications on our computers if we can obtain the same services without the need to do so? This is Cloud Computing for us, the common users. Many of the applications that we used have made the leap to the cloud, where they are totally independent of the operating system that is accessing them, and with a great advantage: that everything is portable. But it is much more than that, and that is that Cloud Computing has radically transformed the business model of both SMEs and large companies, and its strategies to have a new tool that accelerates process innovation and reduce costs.

Keywords: Server, Internet, Applications, Network, Browser. 


\section{Computación en la nube}

Vol. 2, núm. 1., (2018)

Homero Enrique Jinez Jinez; Bolívar Enrique Jinez Sorroza; Jean Pool Jinez Sorroza; Jesus Eliecer Rodríguez Villacis; Washington Antonio Caraguay Ambuludi; Marco Vinicio Sotomayor Sánchez

\section{Introducción.}

El término cloud computing hace referencia a una concepción tecnológica y a un modelo de negocio que reúne ideas tan diversas como el almacenamiento de información, las comunicaciones entre ordenadores, la provisión de servicios o las metodologías de desarrollo de aplicaciones, todo ello bajo el mismo concepto: todo ocurre en la nube. (Martín, 2014)

Tratando de engranar todas estas cuestiones con vistas a la definición de qué es el cloud compunting, quizá debiéramos detenernos un instante y definir el concepto de nube o, para ser más exactos, de internet. Internet, definida de una manera deliberadamente simple, es un conjunto de ordenadores, distribuidos por el mundo y unidos por una tupida malla de comunicaciones, que ofrece espacios de información a todo el que tenga acceso. El acceso a la información que nos ofrecen los ordenadores que componen Internet es "transparente", es decir, no es relevante para el usuario el lugar en el que está alojada físicamente la información. De ahí que Internet se represente de una manera universal, como una nube a la que se accede en busca de información y servicios. (Filipp, 2013)

No todo lo que ocurre en Internet es cloud computing; Internet es un universo que, básicamente, ofrece dos cosas: publicación de información y oferta de servicios. Se puede afirmar que la mera publicación de información no forma parte del modelo de cloud computing, así que, con esto, obtenemos una primera frontera que separa lo que está dentro de nuestro ejercicio de definición de aquello que no lo está. Centrémonos por lo tanto en los servicios. (Martín, 2014) 
Homero Enrique Jinez Jinez; Bolívar Enrique Jinez Sorroza; Jean Pool Jinez Sorroza; Jesus Eliecer Rodríguez Villacis; Washington Antonio Caraguay Ambuludi; Marco Vinicio Sotomayor

Sánchez

\section{No todo lo que ocurre en Internet es 'cloud computing"}

Internet es también un gran mercado de servicios de diversa naturaleza y formato que podríamos dividir en dos grandes grupos, en base al uso que se le da en la red: los servicios que utilizan la red como canal y los que se encuentran en la red y le ofrecen recursos propios. Respecto a los primeros, pensemos en un banco que ofrece sus servicios transaccionales, oficinas virtuales de atención al cliente, canales de venta o subasta... En realidad la utilidad de internet en estos procesos no es sino un mero canal de comunicación. Estos servicios no se consideran cloud computing. (Martín, 2014)

En cuanto a los servicios que se encuentran en la red y le ofrecen recursos propios, destacan los servicios de hosting que nos permiten guardar información fuera de nuestros ordenadores, es decir, en servidores que están en la nube y a los que podemos acceder a través una red de comunicaciones. Otro ejemplo sería el servicio de correo electrónico, en este caso todo, tanto la aplicación que utilizamos como los datos que intercambiamos con nuestros destinatarios, están almacenados en la nube. Estos servicios sí pueden considerarse cloud computing. (Martín, 2014)

Las grandes empresas comenzaron a utilizar este término para referirse a aquellos servicios alojados en la red. De hecho esa es la primera cosa que a la mayoría de nosotros se nos viene a la cabeza sobre "Cloud Computing". Por ello, podemos decir que la palabra nube sería equivalente a lo que conocemos como Internet. (Torres, 2011) 


\section{Computación en la nube}

Vol. 2, núm. 1., (2018)

Homero Enrique Jinez Jinez; Bolívar Enrique Jinez Sorroza; Jean Pool Jinez Sorroza; Jesus Eliecer Rodríguez Villacis; Washington Antonio Caraguay Ambuludi; Marco Vinicio Sotomayor Sánchez

\section{Materiales y métodos.}

La metodología empleada en la presente investigación es una revisión bibliográfica documental de tipo no experimental. En vista que se utilizó como método de investigación diferentes artículos y trabajos de investigación alojados en páginas web, referente a la temática del empleo de elementos informáticos dentro de los procesos administrativos.

\section{Resultados.}

Podemos considerar tres tipologías básicas de servicios que constituyen el modelo de negocio del cloud compuntig y que, dentro del vocabulario informático han venido en llamarse la generación “As a service” (IaaS, PaaS, SaaS).

Para la definición de estos términos seguiremos el camino que siguen los datos de nuestros ordenadores hacia la nube, comenzando por los servicios más vinculados a las máquinas (hardware) hasta llegar a los de naturaleza más lógica (software) pasando por los que hacen posible estos últimos (herramientas de desarrollo). (Martín, 2014)

Ofrecer al cliente espacio de almacenamiento o capacidad de procesamiento en sus servidores. Así el usuario tendrá a su disposición "un disco duro de capacidad ilimitada” y un procesador de rendimiento casi infinito, solo restringido a su capacidad económica de contratación del servicio. Este servicio se basa en el acceso al uso de hardware radicado en la nube. (Martín, 2014) 


\section{Computación en la nube}

Vol. 2, núm. 1., (2018)

Homero Enrique Jinez Jinez; Bolívar Enrique Jinez Sorroza; Jean Pool Jinez Sorroza; Jesus Eliecer Rodríguez Villacis; Washington Antonio Caraguay Ambuludi; Marco Vinicio Sotomayor Sánchez

El servicio de Plataforma pone a disposición de los usuarios herramientas para la realización de desarrollos informáticos, de manera que aquellos pueden construir sus aplicaciones o piezas de software sin necesidad de adquirir e implantar en sus ordenadores locales dichas herramientas. Este servicio tiene dos claras ventajas para el desarrollador de aplicaciones: no tiene que adquirir las costosas licencias para desarrollo de las herramientas de mercado y, por otra parte, el proveedor de servicios se encarga de que dichas herramientas estén en óptima situación de mantenimiento. (Martín, 2014)

\section{Ventajas del Cloud Computing}

Vale acotar, Uno de los grandes beneficios que aporta el Cloud Computing es la posibilidad de aumentar los recursos de acuerdo con las necesidades. Este es uno de los conceptos que resulta más atractivo para empresas que trabajan con picos de demanda estacionales. La tecnología permite que el usuario aumente o disminuya de forma sencilla y ágil la cantidad de recursos contratados sin necesidad de modificar contratos. En general, las propias empresas usuarias del servicio logran administrar este cambio por medio de tableros de control en línea. Por ejemplo: si una empresa de e-commerce tiene un flujo de atención al cliente mayor en un determinado período, es posible aumentar el número de servidores para cubrir esta demanda. Cuando esto no sea más necesario, sólo hay que regresar a la cantidad normal de servidores de manera rápida y sin problemas de adaptación. (Comstor, 2014)

\section{Accesibilidad global}




\section{Computación en la nube}

Vol. 2, núm. 1., (2018)

Homero Enrique Jinez Jinez; Bolívar Enrique Jinez Sorroza; Jean Pool Jinez Sorroza; Jesus Eliecer Rodríguez Villacis; Washington Antonio Caraguay Ambuludi; Marco Vinicio Sotomayor Sánchez

A través de Cloud Computing es posible poner a disposición una sola aplicación para miles de usuarios conectados. Para acceder a los datos disponibles "en la nube" desde cualquier lugar, lo único que se necesita es tener una conexión de Internet. Esto es muy importante para empresas que tienen profesionales dispersos geográficamente o que permanecen mucho tiempo fuera de la oficina. Imagina la eficiencia que esto aporta al equipo de ventas de una empresa multinacional, por ejemplo. Independientemente del lugar en que se encuentre, el vendedor tiene acceso total a toda la información que requiere para una negociación. (Comstor, 2014)

\section{Mayor productividad}

Cloud Computing permite que las personas dediquen más tiempo a trabajar en actividades que generen valor para la empresa que cuestiones meramente operativas. Esto permite utilizar mejor el equipo de trabajo, reducir el tiempo de ejecución y aumentar la productividad. Piensa en los beneficios que obtiene un equipo de TI por estar totalmente enfocado en el desarrollo de soluciones para determinado proyecto, sin tener que preocuparse por la instalación de hardware y software, implementación de sistemas, instalación de data centers u otras tareas de este tipo. Mejor Colaboración entre equipos de trabajo. (Comstor, 2014)

\section{Fácil gestión y mantenimiento}

En servicios de Cloud Computing toda la infraestructura del servicio utilizado, así como su actualización y mantenimiento, queda a cargo del proveedor del servicio. Pero también es posible que la empresa gestione y monitoree sus dispositivos de forma remota, además de 


\section{Computación en la nube}

Vol. 2, núm. 1., (2018)

Homero Enrique Jinez Jinez; Bolívar Enrique Jinez Sorroza; Jean Pool Jinez Sorroza; Jesus Eliecer Rodríguez Villacis; Washington Antonio Caraguay Ambuludi; Marco Vinicio Sotomayor Sánchez

administrar fallas, vulnerabilidades, accesos de usuarios, asignación de servidores, tiempo de uso y mucho más. Todo esto por medio de una sola interfaz. (Comstor, 2014)

\section{Desventajas del Cloud Computing}

En general, entre las mayores ventajas de la nube se destaca su seguridad. Si bien es cierto que el nivel de seguridad es alto hay que apuntar que el cloud computing tiene también ciertas desventajas o posibles fallos que pueden ocurrir. (Filipp, 2013)

La más renombrada de las desventajas es la vulnerabilidad del acceso a la información. Empecemos primero por comprender que desplazar nuestra información fuera del alcance físico ya es abrumador y si a esto le sumamos el fácil acceso a ella, el "miedo" nos toma como presa. Para compensar un poco este temor que tiene el cliente, las compañías tienen grandes sistemas de alta seguridad para mantener los datos a salvo. (Team - Doble Group, 2015)

Asimismo, otras desventajas o aspectos negativos que han causado muchas dudas en las organizaciones a la hora de adoptarlo, vamos a mencionar los principales:

Seguridad

Es la principal preocupación que existe con respecto a esta solución tecnológica y es al mismo tiempo el principal desafío al que se enfrentan las empresas proveedoras del servicio que 


\section{Computación en la nube}

Vol. 2, núm. 1., (2018)

Homero Enrique Jinez Jinez; Bolívar Enrique Jinez Sorroza; Jean Pool Jinez Sorroza; Jesus Eliecer Rodríguez Villacis; Washington Antonio Caraguay Ambuludi; Marco Vinicio Sotomayor Sánchez

deben mejorar sus sistemas de encriptación y cifrado continuamente para proteger los datos. El compromiso de las compañías de Cloud Computing es brindar una infraestructura robusta con el mayor nivel de seguridad y confiabilidad posible, pero debemos recordar que este sistema implica necesariamente que la información y los datos almacenados en los servidores externos tengan que estar disponibles en línea para poder acceder a ellos, lo que significa inevitablemente que al circular por internet la información es susceptible de ser interceptada o modificada por terceros que se especializan en ese tipo de delitos informáticos. (Santanas, 2013)

\section{Privacidad}

La privacidad de los datos y la información es uno de los aspectos más sensibles en una organización, los próximos desarrollos, los proyectos a futuro o el lanzamiento de nuevos productos y servicios son ejemplos de asuntos clave para el desempeño de una compañía que al estar alojados en los servidores del proveedor de Cloud Computing evidentemente dejan de ser conocidos sólo por la organización dueña de ellos. (Santanas, 2013)

La confianza es la premisa básica cuando se le entregan los datos al proveedor para su almacenamiento, al hacerlo estamos seguros que las garantías que se nos dan evitarán cualquier tipo de filtración o fuga de información, pero debemos ser conscientes que al adoptar el servicio la información deja de estar exclusivamente en nuestro poder y que en un mundo completamente abierto como el de internet todo puede pasar, aún más cuando la privacidad es uno de los aspectos más polémicos y discutidos en la red puesto que nunca va estar garantizada al cien por ciento. (Santanas, 2013) 


\section{Computación en la nube}

Vol. 2, núm. 1., (2018)

Homero Enrique Jinez Jinez; Bolívar Enrique Jinez Sorroza; Jean Pool Jinez Sorroza; Jesus Eliecer Rodríguez Villacis; Washington Antonio Caraguay Ambuludi; Marco Vinicio Sotomayor Sánchez

\section{Conectividad}

Entre los beneficios del Cloud Computing mencionábamos la facilidad de acceso a los datos mediante una conexión a internet y un dispositivo apto para ello, pero ésta es al mismo tiempo una gran desventaja puesto que la posibilidad de acceso a la información depende directamente de la calidad y disponibilidad del servicio que preste nuestro proveedor de internet (ISP). (Santanas, 2013)

Al estar alojados en servidores externos, los datos no están físicamente en nuestra empresa y sólo se pueden gestionar en la nube por medio de la conexión a internet, que cuando no funcione, situación que puede darse en cualquier momento por muchos factores, podrían provocar problemas o fallas operativas graves si necesitamos la información urgentemente, esto quiere decir que a pesar que los datos están siempre en línea su acceso depende nuevamente de un tercero, la compañía de internet, y no de la organización ni del proveedor de Cloud Computing. (Santanas, 2013)

\section{Conclusiones.}

A la hora de la elección de un servicio de almacenamiento en la nube, debemos de analizar las necesidades de almacenamiento que tenemos y la utilidad que le vamos a dar. Estos factores son determinantes para decantarnos por uno u otro servicio. (fiie-sdan, 2012)

Todos cumplen con el objetivo principal: procesar y almacenar en la nube todo el contenido que desee el usuario hasta el límite de su cuenta, de modo que podamos acceder a él desde cualquier lugar con conexión a Internet, sin la necesidad de depender de un dispositivo. 


\section{Computación en la nube}

Vol. 2, núm. 1., (2018)

Homero Enrique Jinez Jinez; Bolívar Enrique Jinez Sorroza; Jean Pool Jinez Sorroza; Jesus Eliecer Rodríguez Villacis; Washington Antonio Caraguay Ambuludi; Marco Vinicio Sotomayor Sánchez

Hemos podido comprobar que cada servicio tiene sus propias características, sus ventajas y sus inconvenientes. (fiie-sdan, 2012)

Para los usuarios cuya única finalidad sea el alojamiento de documentación y la variedad de servicios y opciones que le brinda a los usuarios.(fiie-sdan, 2012)

\section{Recomendaciones.}

Cada vez son más los negocios que están apostando por la tecnología del Cloud Computing virtualizando su infraestructura y moviendo sus aplicaciones en formato de servicio IaaS sin del todo saber qué ventajas les puede aportar realmente la nube. (ACKSTORM, 2014)

Sin embargo, según estudios recientes de analistas especializados en el sector, la tendencia del Cloud Computing para este año 2014 es la de la migración al PaaS. (ACKSTORM, 2014)

La rapidez y soporte en el desarrollo de las aplicaciones que supone el PaaS, junto a su agilidad, modalidad de pago por uso y escalabilidad son argumentos necesarios para apostar por esta modalidad de Cloud. De todas maneras, se ha detectado que las dudas y miedos sobre la efectividad que supone migrar al Cloud continúan siendo una importante barrera para las empresas. (ACKSTORM, 2014)

1. Con el fin de evitar intrusiones, recomendamos realizar una prueba de vulnerabilidades para identificar los fallos de seguridad que puedan sufrir los sistemas y así proceder a una migración eficiente y segura. 


\section{Computación en la nube}

Vol. 2, núm. 1., (2018)

Homero Enrique Jinez Jinez; Bolívar Enrique Jinez Sorroza; Jean Pool Jinez Sorroza; Jesus Eliecer Rodríguez Villacis; Washington Antonio Caraguay Ambuludi; Marco Vinicio Sotomayor

Sánchez

2. Asesórate con profesionales expertos en migraciones y virtualizaciones de sistemas para que diseñen e implementen la solución que más se adapte a tus necesidades.

3. La automatización de los procesos de análisis y testeo de código para reducir costes es una metodología muy eficiente en estos casos. Además, esto reduce a la vez el margen de error humano. (ACKSTORM, 2014)

\section{Bibliografía.}

ACKSTORM. (24 de Julio de 2014). dataprix.com. Obtenido de dataprix.com: http://www.dataprix.com/noticias-it/cloud-computing/recomendaciones-buenamigracion-cloud

Comstor, C. (06 de Mayo de 2014). Canal Comstor. Obtenido de Canal Comstor: http://blogmexico.comstor.com/principales-beneficios-de-cloud-computing

fiie-sdan. (18 de Diciembre de 2012). jprm-fiie-sdan. Obtenido de jprm-fiie-sdan: http://jprmfiie-sdan.blogspot.com/2012/12/4-conclusion.html

Filipp, S. (18 de Abril de 2013). internetlab.es. Obtenido de internetlab.es: https://www.internetlab.es/post/2123/desventajas-cloud-computing/

Martín, E. (Diciembre de 2014). ticbeat. Obtenido de ticbeat: http://www.ticbeat.com/cloud/quees-cloud-computing-definicion-concepto-para-neofitos/

Santanas, C. (06 de Septiembre de 2013). blog.acsendo.com. Obtenido de blog.acsendo.com: http://blog.acsendo.com/cloud-computing-desventajas-e-inconvenientes/

Team - Doble Group. (04 de Septiembre de 2015). doblegroup.com. Obtenido de doblegroup.com: https://doblegroup.com/es/beneficios-de-cloud-computing-ventajas-ydesventajas/

Torres, G. (20 de Enero de 2011). desarrolloweb. Obtenido de desarrolloweb: https://desarrolloweb.com/articulos/que-es-cloud-computing.html 